\title{
Failure prediction of T-peel adhesive joints by different cohesive laws and modelling approaches
}

\author{
Alessandro Pirondi \\ University of Parma, Department of Industrial Engineering, Parco Area delle Scienze, 181/ A - 43100 Parma, Italy \\ alessandro.pirondi@unipr.it
}

RIASSUNTO. In questo articolo si è simulato mediante il modello di zona coesiva il cedimento di un giunto Tpeel incollato. Gli aderendi sono lamiere di acciaio Fe360 e sono unite mediante l'adesivo strutturale Loctite Multibond 330.

I parametri del modello di zona coesiva sono stati calibrati sulla base di esperimenti di frattura condotti in precedenza su provini Double Cantilever Beam (DCB) incollati con il medesimo adesivo. La simulazione è stata condotta utilizzando il software di analisi ad elementi finiti ABAQUS, sviluppando modelli 2-D. Il cedimento avviene in uno strato modellato utilizzando elementi di tipo coesivo disponibili nel software. L'analisi è volta ad individuare l'influenza di: i) differenti formulazioni della legge coesiva, ii) la modellazione o meno dello strato di adesivo con le sue proprietà elasto-plastiche.

ABSTRACT. In this work, Cohesive Zone Modelling (CZM) was used to simulate failure of T-peel bonded joints with $1.5 \mathrm{~mm}$ thick adherends, respectively, bonded toghether with Loctite Multibond 330 adhesive. The fracture toughness and load-opening behaviour recorded in previous experiments on bonded Double Cantilever Beam (DCB) specimens were taken as reference to calibrate CZM parameters. Two-dimensional models were analysed using the FE code ABAQUS. The failing interface was modeled with the cohesive elements available in this software. The influence of: i) different cohesive law shapes, ii) modeling the presence of the adhesive layer explicitly, was studied.

KEYwORDS. Adhesive joints, Fracture, Cohesive zone modeling.

\section{INTRODUCTION}

$\mathrm{T}$ he use of adhesive joining in civil, aerospace and mechanical constructions has considerably increased in the last decades thanks to the advantages over traditional joining techniques such as: i) ability to join dissimilar materials, ii) stress distribution over a wider area, iii) potentially lower weight. However, joint fabrication procedures and component service loads may introduce or initiate defects, whose evolution will control the performance and the reliability of the bonded joint. In those cases, Fracture Mechanics (FM) can be used to assess the structural integrity of a bonded joint [1]. The FM approach consists in the comparison of a parameter, function of load and geometry of the cracked body (for example the strain energy release rate, $G$ ), with the fracture resistance $\left(G_{c}\right)$. The simulation of fracture therefore requires to implement a criterion that triggers propagation when $G=G_{c}$.

An attractive way to simulate the effect of a defect on joint strength is to incorporate a model of the rupture process (i.e. the criterion to trigger propagation). In particular, the fracture of bonded joints has been successfully simulated using the Cohesive Zone Model (CZM) in [2-7]. According to this approach, the zone in front of the physical crack tip opens and then tears progressively apart following a given traction-separation behaviour.

Although straightforward methods to evaluate experimentally CZM parameters in adhesive joints have been recently presented [8], questions on the physical meaning or, in other words, on the transferability of the parameters to joint geometries different from the one from they were extracted is still an open issue. In particular, studies have been carried 
out about the influence of the thickness of the adhesive layer and of the adherends [9-11] that can be seen also as the evaluation of different degrees of constraint. The constraint effect in homogenous materials is well known to arise from the out-of-plane width and from the in-plane interaction of the crack with the boundaries. In the case of a crack in an adhesive joint, the adhesive layer thickness defines the distance of the boundaries (i.e. the adherends) from the crack tip. The flexibility of the boundaries plays of course also an important role in the constraint imparted to the crack.

In a previous work [12], 2D CZM analyses were carried out to simulate failure of T-peel bonded joints) with 1.5 and $3 \mathrm{~mm}$ thick adherends, respectively. A trapezoidal cohesive law was used and a CZ was introduced using nonlinear springs. The fracture toughness and load-opening behaviour recorded in experiments on bonded DCB specimens [13] were taken as reference to calibrate CZM parameters.

This work is aimed at extending those results. The failing interface is modeled with the cohesive elements available in this software. The influence of: i) different cohesive law shapes, ii) modelling the presence of the adhesive layer explicitly, is studied.

\section{EXPERIMENTAL}

\section{Materials}

7 he DCB aluminum adherends $(\mathrm{E}=70 \mathrm{GPa}, \mathrm{v}=0.3)$ were bonded with Loctite 330, a modified methacrilate ester supplied as viscous paste plus activator. Loctite 330 is a structural adhesive suitable for bonding of metals, wood, ceramics and plastics. Its advantage with respect to other structural adhesives is that it does not require specific surface preparation (only degreasing) and the joint can be handled after five minutes, allowing for higher production rates. Shear and tensile strength declared by the supplier are about 20MPa. The tensile behavior of the adhesive was modeled as a power law:

$$
\begin{array}{ll}
\sigma=\mathrm{E} \varepsilon & \sigma \leq \sigma_{0} \\
\sigma=\sigma_{0} *\left(\varepsilon / \varepsilon_{0}\right)^{\mathrm{n}} & \sigma>\sigma_{0}
\end{array}
$$

where $\mathrm{E}=878 \mathrm{MPa}, \sigma_{0}=4.8 \mathrm{MPa}, \mathrm{n}=0.44$ and $\varepsilon_{0}=\sigma_{0} / \mathrm{E}$ were determined by tensile testing of bulk adhesive [13]. The Poisson's ratio was 0.15 .

The T-peel joints were made of italian standard UNI Fe 360 unalloyed steel, whose properties are typically E=210GPa, $v=0.3, \sigma_{\mathrm{u}}=360 \mathrm{MPa}, \sigma_{0}=240 \mathrm{MPa}$.

\section{DCB tests}

The specimen dimensions are shown in Fig. 1. A four-step procedure was used for surface preparation of the substrates before bonding: i) polishing with sandpaper, ii) degreasing with acetone, iii) rinsing in hot water, iv) drying. The procedure was applied twice, initially using a coarse sandpaper and then a fine sandpaper.

The recommended bondline thickness for Multibond $330^{\circledR}$ ranges between 0.05 to $0.5 \mathrm{~mm}$. In all the experiments reported here, a thickness of $0.25 \mathrm{~mm}$ was adopted. Bond thickness control during specimen preparation was achieved placing two controlled-thickness copper shims between the adherends (see Fig. 1a). A 0.05-mm-thick Teflon tape was placed just before bonding at mid-thickness of the adhesive layer to obtain a cohesive crack-like defect.

The test were performed after at least a 24-hrs curing at room temperature, that is the minimum time to fully develop the mechanical strength according to the datasheet of the adhesive manufacturer.
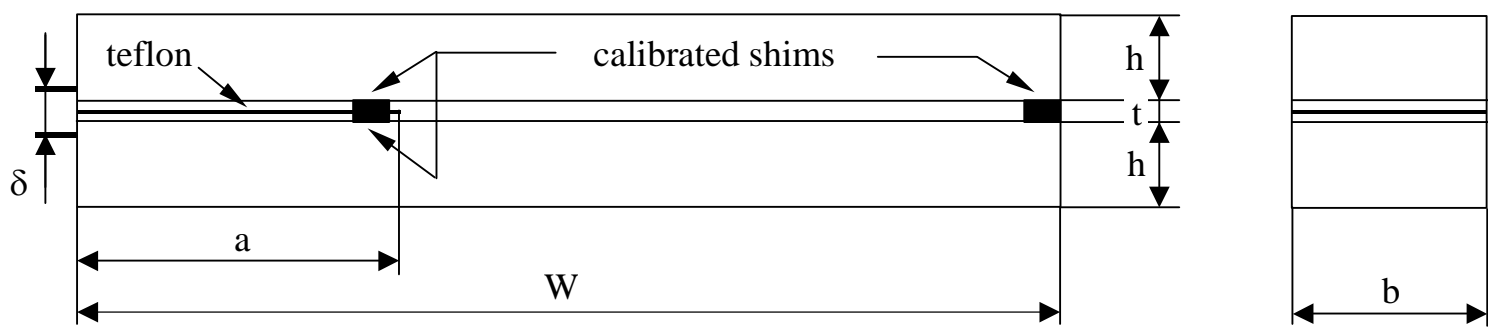

$\mathrm{a}_{0}=40 \mathrm{~mm}$

$\mathrm{W}=120 \mathrm{~mm}$

$\mathrm{h}=15 \mathrm{~mm}$

$\mathrm{t}=0.3 \mathrm{~mm}$

$\mathrm{b}=30 \mathrm{~mm}$

Figure 1: Outline of the DCB joint. 
To account for the elasticity of the adhesive, the DCB specimen can be modeled as a beam on an elastic foundation, where the foundation modulus, $\mathrm{k}$, will depend on the elastic constants of the adhesive (i.e. $\mathrm{E}_{\mathrm{a}}$ and $\mathrm{v}_{\mathrm{a}}$ ) and on the bondline thickness, t. The solution developed in [14] neglecting shear contributions was therefore considered. The load line compliance is given by the following equation:

$$
\mathrm{C}=\frac{\Delta}{\mathrm{P}}=\frac{2 \lambda_{\sigma} \mathrm{t}}{\mathrm{E}_{\mathrm{a}}^{\prime} \mathrm{b}}\left[1+2\left(\lambda_{\sigma} \mathrm{a}\right)+2\left(\lambda_{\sigma} \mathrm{a}\right)^{2}+\frac{2}{3}\left(\lambda_{\sigma} \mathrm{a}\right)^{3}\right]
$$

where $E_{a}^{\prime}=E_{a} /\left(1-v_{a}^{2}\right)$ is plane strain modulus of the adhesive and $\lambda_{\sigma}^{4}=\frac{4 k}{E I}=\frac{6}{h^{3} t} \frac{E_{a}^{\prime}}{E}$. The strain energy release rate $G$ is given by

$$
G=\frac{P^{2}}{2 b}\left(\frac{\partial C}{\partial a}\right)=\frac{P^{2} a^{2}}{b E I}\left(1+\frac{1}{\lambda_{\sigma} a}\right)^{2}
$$

For the present DCB specimen the term $\left(\lambda_{\sigma} \mathrm{a}\right)^{-1}$ is about 0.25 .

\section{T-peel tests}

A series of tensile tests was conducted at the Polytechnic of Turin [15] on UNI Fe360 construction steel T-peel joints (Fig. 2).

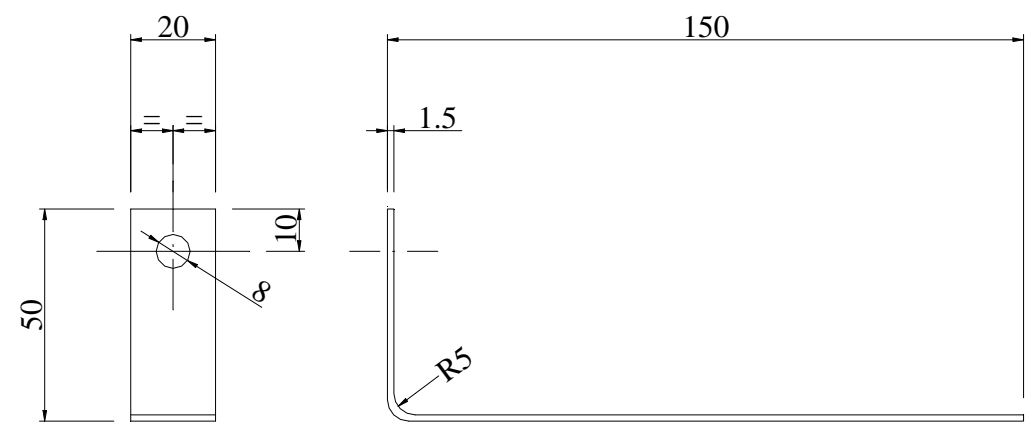

Figure 2: Outline of half of the 1.5mm-thick T-peel joint tested in [15].

A metal sheet thickness of $1.5 \mathrm{~mm}$ was investigated. The adhesive used is again the Loctite $330^{\circledR}$. A bondline thickness of $0.1 \mathrm{~mm}$ was obtained in this case. Displacement-controlled tests were conducted up to partial or complete separation of the two halves.

\section{MODELLING}

\section{Fe models}

he 2D FE models corresponding to DCB and T-peel joints are shown in Figs. 3a-b. Symmetry conditions with respect to the bondline have been applied. Plane stress, eight-noded isoparametric elements have been used to simulate the metal adherend.

The cohesive zone has been modelled with the cohesive elements available in the FE code ABAQUS. A convergence analysis was preliminarly conducted to determine the appropriate element size. The presence of the adhesive layer was either included in the cohesive zone (Adherend + Cohesive Zone, ACZ) or explicitly modelled with four-noded, hybrid, plane strain elements (Adherend + Adhesive + Cohesive Zone, AACZ), as done for example in [11]. The number of elements along the thickness of the bond was defined so far according to the indications given in [16]. In the case of the T-peel joint, different extensions of the adhesive layer at the fillet were simulated, since they were not known in detail. 


\section{Cohesive law}

Three different kind of cohesive laws were accounted for: i) trapezoidal, ii) triangular and iii) exponential as shown qualitatively in Fig. 4. The relationship between the parameters of the laws is:

i) $\Gamma_{0}=\frac{1}{2} \sigma_{m} \delta_{c}\left[1+c_{2}-c_{1}\right]$

ii) $\Gamma_{0}=\frac{1}{2} \sigma_{m} \delta_{c}$

iii) $\Gamma_{0}=\sigma_{m}\left(\delta_{c}-\delta_{1}\right)\left(1-\frac{1}{1-e^{-\alpha}}+\frac{1}{\alpha}\right)$

where $\mathrm{c}_{1}=\delta_{1} / \delta_{\mathrm{c}}$ e $\mathrm{c}_{2}=\delta_{2} / \delta_{\mathrm{c}}$. The cohesive energy $\Gamma_{0}$ was taken equal to the fracture toughness measured in [11], that is $\Gamma_{0}=\mathrm{G}_{\mathrm{Ic}}=550 \mathrm{~J} / \mathrm{m}^{2}$ in the case of the ACZ kind of models. The parameters of the different laws were tuned until the peak load of the DCB test was achieved, while keeping also a good correspondance with the overall behaviour.

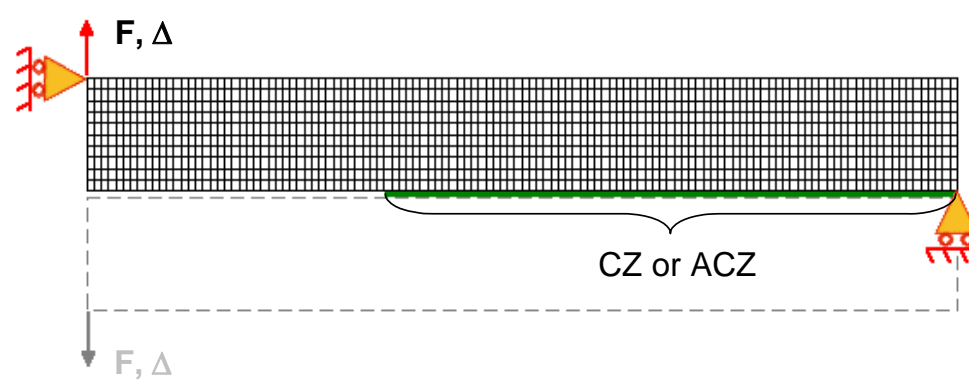

(a)

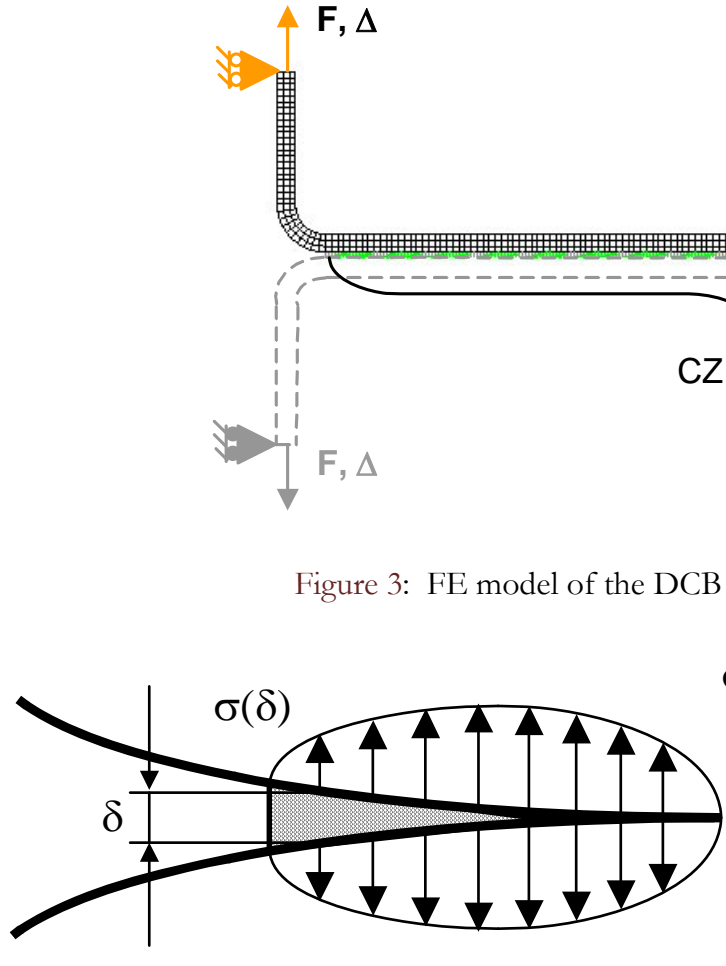

(b)

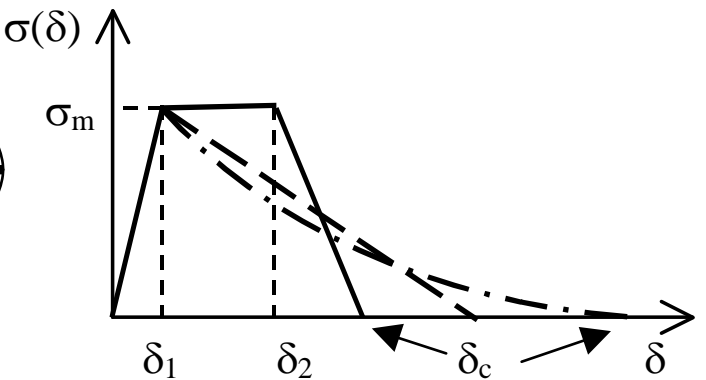

Figure 4: Outline of the cohesive zone traction-separation laws (Eqns. 4 - 6). 


\section{RESULTS}

\section{Tuning of $C Z$ parameters}

7 he first step was the tuning of the DCB-ACZ model on the related experiment performed in [13]. Some parameters were pre-calibrated based on the results shown in a previous work [12]. Therefore, a value of

$\sigma_{\mathrm{m}}=5 \mathrm{MPa}$ was adopted in all of the models and in the case of the trapezoidal law, $\mathrm{c}_{1}=0.2$ and $\mathrm{c}_{2}=0.5$ were taken, as tipically found in the literature. It is visible in Fig. 5 that with such a cohesive law the propagation phase is well matched and the peak load is only slightly overestimated, but initial slope is quite different. Much better results are obtained instead with a triangular law with $\mathrm{c}_{1}=0.01$. A similar agreement (not shown here for the sake of clarity) was found with the trapezoidal law using $\mathrm{c}_{1}=0.01$ and $\mathrm{c}_{2}=0.02$, in fact an almost triangular law. The exponential law was taken with a $\delta_{1}$ equal to the $\delta_{1}$ defined for the triangular law in order to keep the same initial slope. Anyway, this choice resulted in a lower peak load and a different post-failure trend with respect to the triangular law. Specifically, it resembles the results that would be obtained using a lower value of $\sigma_{\mathrm{m}}$. All of the following simulation were therefore conducted using the triangular law, which incidentally is also easier to handle.

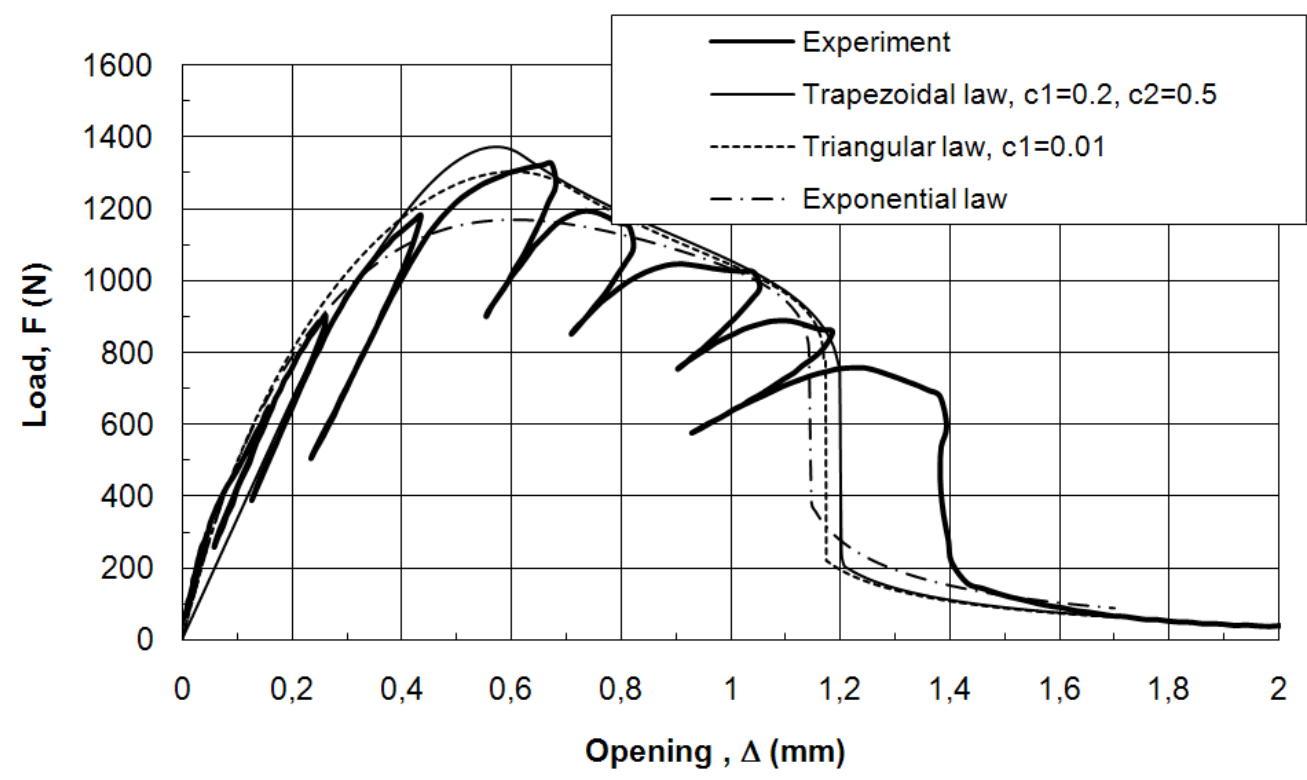

Figure 5: Tuning of ACZ model on DCB experiment.

The value of $\sigma_{\mathrm{m}}=5 \mathrm{MPa}$ outcome from the ACZ tuning is only a bit higher than the yield strength of the adhesive (4.8MPa). The calibration of AACZ parameters was attempted using couples of $\left(\Gamma_{0}, \sigma_{\mathrm{m}}\right)$ lower and higher than the values obtained for the ACZ model, respectively, as shown in [11]. Anyway, since the value of $\sigma_{\mathrm{m}}=5 \mathrm{MPa}$ outcome from the ACZ tuning is only a bit higher than the yield strength of the adhesive $(4.8 \mathrm{MPa})$, plasticity within the adhesive layer is not significant and the AACZ model did not significantly differ from the ACZ.

\section{Simulation of T-peel tests}

The simulation of T-peel tests was conducted using an ACZ model with $\sigma_{\mathrm{m}}=12.5 \mathrm{MPa}$ to take into account the higher adesive layer stiffness of the T-peel joints with respect to the DCB due to the different thickness (i.e. $0.1 \mathrm{~mm}$ against $0.25 \mathrm{~mm}$ ) and $\sigma_{\mathrm{m}}=20.6 \mathrm{MPa}$, to take into account also the higher strength when joining steel (T-peel) instead of aluminum (DCB), as described in the technical datasheet of the adhesive. The results shown in Fig. 6 in the case of a 1.5mmthick Tpeel arm exhibit a good correlation with the experiments in the propagation phase but a very poor match in the loading phase, regarding both the slope and the peak load. On the other hand, it is known [11] that in very thin adhesive layers as in the present case plasticity is strongly confined and due to this, the value of $\sigma_{\mathrm{m}}$ of an ACZ model increases with decresing adhesive layer thickness. 


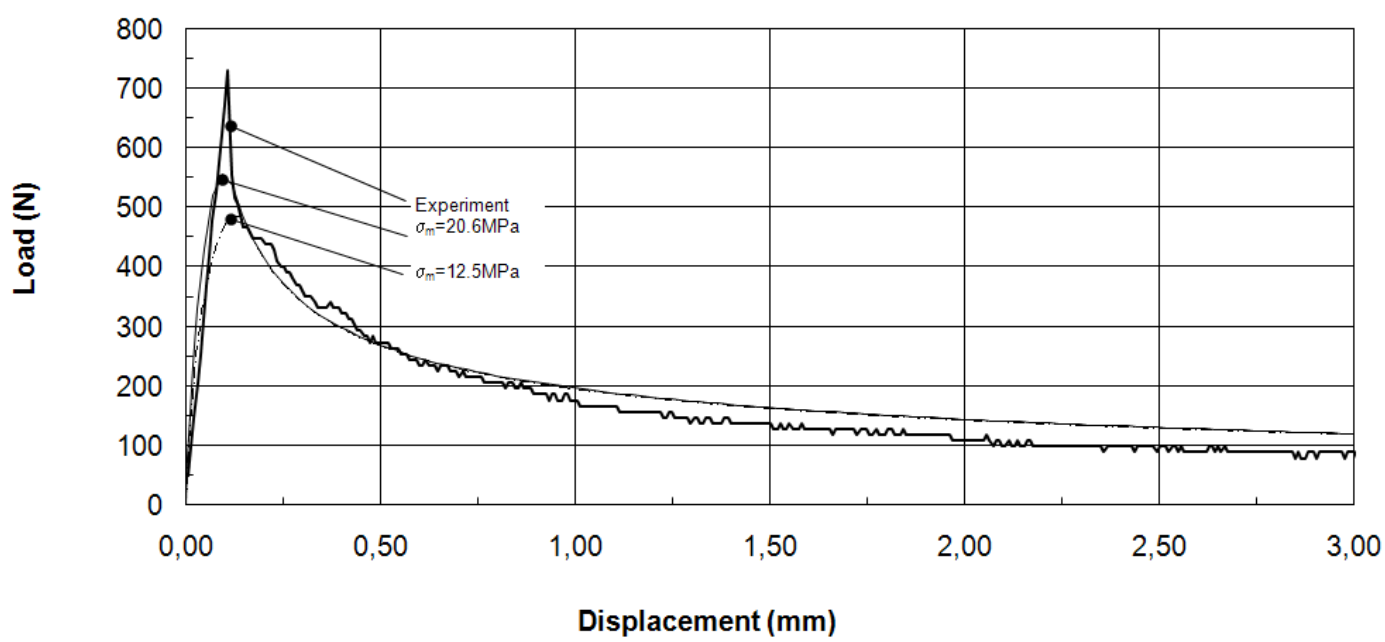

Figure 6: Comparison between experiment and T-peel simulations (ACZ model).

The simulations done with the AACZ kind of model are shown in Fig. 7 in the case of $1.5 \mathrm{~mm}$-thick peel arm. The use of the parameters determined from DCB $\left(\sigma_{\mathrm{m}}=5 \mathrm{MPa}, \Gamma_{0}=550 \mathrm{~J} / \mathrm{m}^{2}\right)$, where ACZ and AACZ were coincident, resulted in a large difference with the experimental behavior.

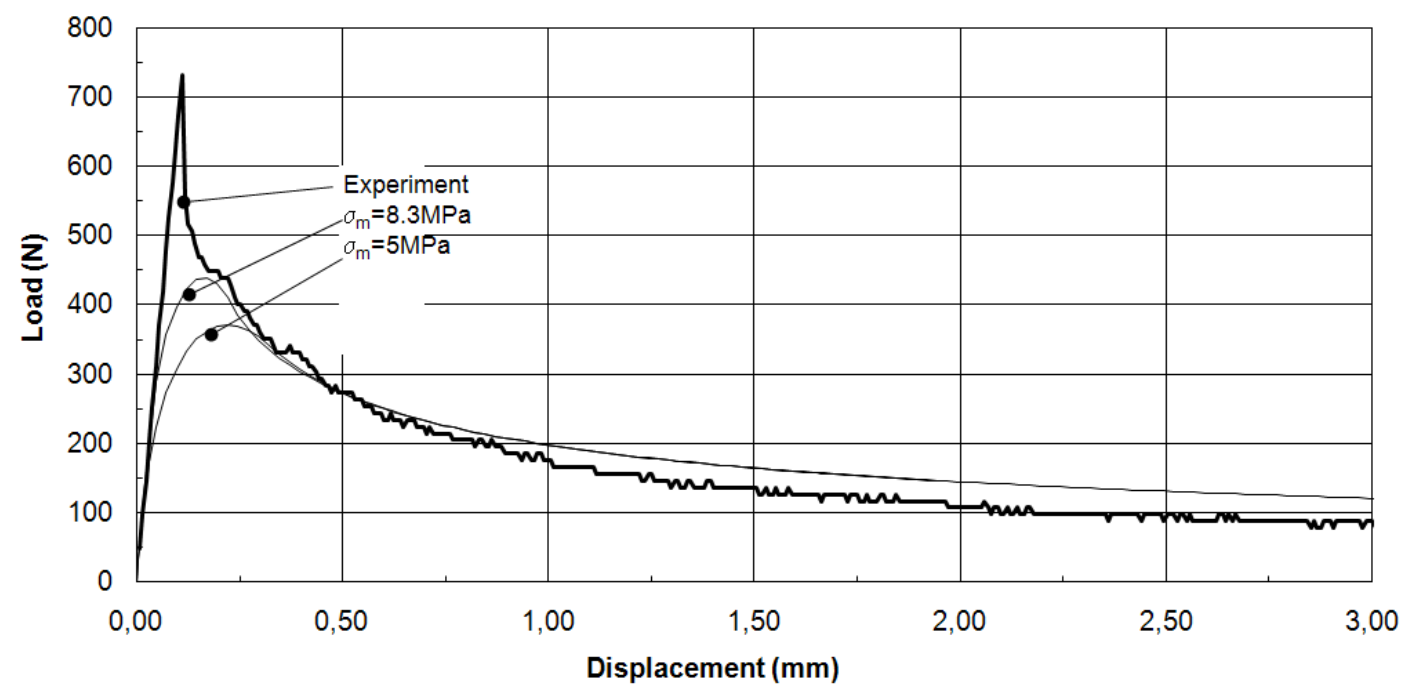

Figure 7: Comparison between experiment and T-peel simulations (AACZ model).

From these analyses, it is evident that CZ parameters may not be the same for the nucleation and propagation of a crack. Besides discrepancies that may arise between the properties of bulk adhesive and joint, and between different joint geometries due to the bonding process, where adhesive and activator cannot be pre-mixed in a fixed ratio, it has been demonstrated that the tearing process is influenced by the local constraint [11].

Therefore, a further analysis has been attempted using AACZ modeling where:

i) the adhesive was extended at the fillet, with the extension length limited by the maximum adhesive thickness that can be polimerized according to the supplier datasheet;

ii) the cohesive strength $\sigma_{\mathrm{m}}$ has been defined as the value of stress in the adhesive normal to the plane of the joint, at the point in the analysis where the uniaxial failure strain was met locally. The distribution of cohesive strength obtained in this way is shown in Fig.8.

The results of the simulation with the cohesive law input from Fig. 8 and $\Gamma_{0}=550 \mathrm{~J} / \mathrm{m}^{2}$ reported in Fig. 9 show a neat increase of the agreement with the experiment concerning the phase up to the maximum load, while the crack propagation phase is matched well as in the previous analyses. This fact confirms that the crack propagation may be reproduced quite easily tuning the $\mathrm{CZ}$ parameters on standard fracture experiments, while the nucleation requires specific 
parameters, possibly dependent on the local stress state. In the specific case of the T-peel test it is important also to know the real fillet dimensions.

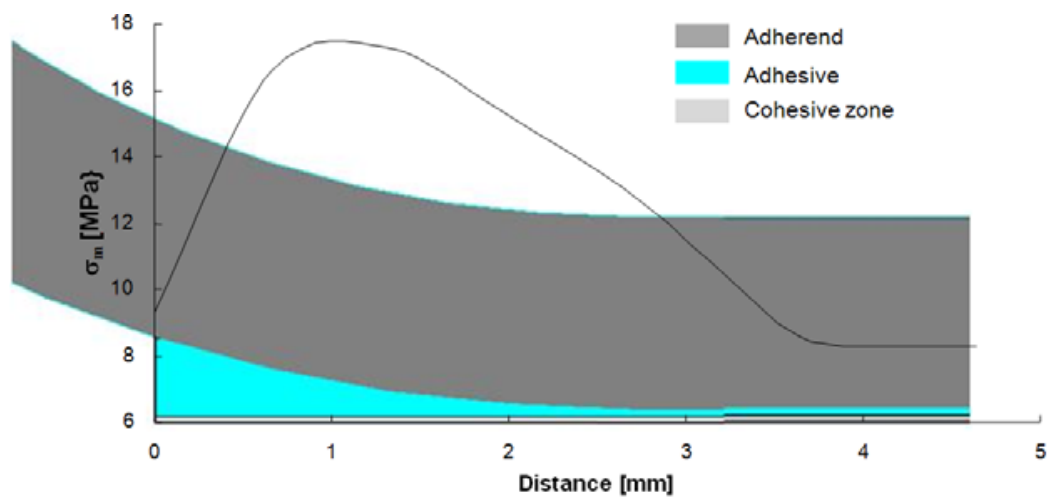

Figure 8: Distribution of cohesive strength (AACZ model) obtained as the value of stress in the adhesive normal to the plane of the joint, at the point in the analysis where the uniaxial failure strain was met locally.

\section{CONCLUSIONS}

7 he fracture of adhesively bonded T-peel joints was simulated using a Cohesive Zone Model. The CZ parameters were calibrated on fracture tests conducted on a DCB joint bonded with the same adhesive as the T-peels. The explicit modelling of the adhesive layer (AACZ) did not give in this case any significant variation of the parameters with respect to the simpler model where the adhesive was included in the CZ behaviour (ACZ). The T-peel test was simulated first using the set of parameters determined from DCB and the ACZ approach, after a partial recalibration to account for the different thickness of the bondline and for the different metal joined. The results showed a good agreement between experiments and simulations with respect to the propagation phase but the peak load is underestimated and the stiffness is dependent on the modelling of the adhesive at the arm fillet. The simulation with the AACZ approach did not give better results using the same set of parameters. A much better agreement was found using a cohesive strength $\sigma_{\mathrm{m}}$ defined as the value of stress in the adhesive normal to the plane of the joint, at the point in the analysis where the uniaxial failure strain was met locally. This means that the crack propagation may be reproduced quite easily tuning the $\mathrm{CZ}$ parameters on standard fracture experiments, while the nucleation requires specific parameters, possibly dependent on the local stress state.

\section{ACKNOWLEDGEMENTS}

7 he author gratefully acknowledge Prof. M. Rossetto, Polytechnic of Turin, Italy, for the experimental data of Tpeel joints.

\section{REFERENCES}

[1] A. J. Kinloch, , Adhesion and Adhesives, Chapman and Hall, London, UK, (1986).

[2] J. W. Hutchinson, A. G. Evans, Acta Mater., 48 (2000) 125.

[3] I. Mohammed, K.M. Liechti, J. Mech. Phys. Solids, 48 (2000) 735.

[4] Q.D. Yang, M.D.Thouless, S.M. Ward, J. Mech. Phys. Solids, 47 (1999) 1337.

[5] W. G. Knauss, G. U. Losi, J. Appl. Mech., 60 (1993) 793.

[6] H. Hadavinia, A.J. Kinloch, J.G. Williams, in Adv. in Fract. and Damage Mech. II, M. Guagliano and M.H. Aliabadi eds., Hoggar, Geneva, (2001) 445.

[7] B. F. Sorensen, Acta Mater., 50 (2002) 1053.

[8] B. F. Sorensen, T. K. Jacobsen, Eng. Fract. Mech., 70 (2003) 1841. 
[9] I. Georgiou, H. Hadavinia, A. Ivankovic, A. J. Kinloch, V. Tropsa, J. G. Williams, J. Adhesion, 79 (2003) 239.

[10] B. R. K. Blackman, H. Hadavinia, A. J. Kinloch, J. G. Williams, Int. J. Fract., 119 (2003) 25.

[11] T. Pardoen, T. Ferracin, C. M. Landis, F. Delannay, J. Mech. Phys. Solids, 53 (2005) 1951.

[12] A. Pirondi, Proc. ECF 15, Stockolm, Sweden (2004).

[13] A. Pirondi, G.Nicoletto, Proc. IGF 2000, Bari, Italy, (2000).

[14] S. Krenk, , Eng. Fract. Mech., 43-4 (1992) 549.

[15] M. Rossetto, Private communication, Polytechnic of Turin, Turin, Italy (2003).

[16] K. S. Madhusudhana, R. Narashiman, Eng. Fract. Mech., 69 (2002) 865. 